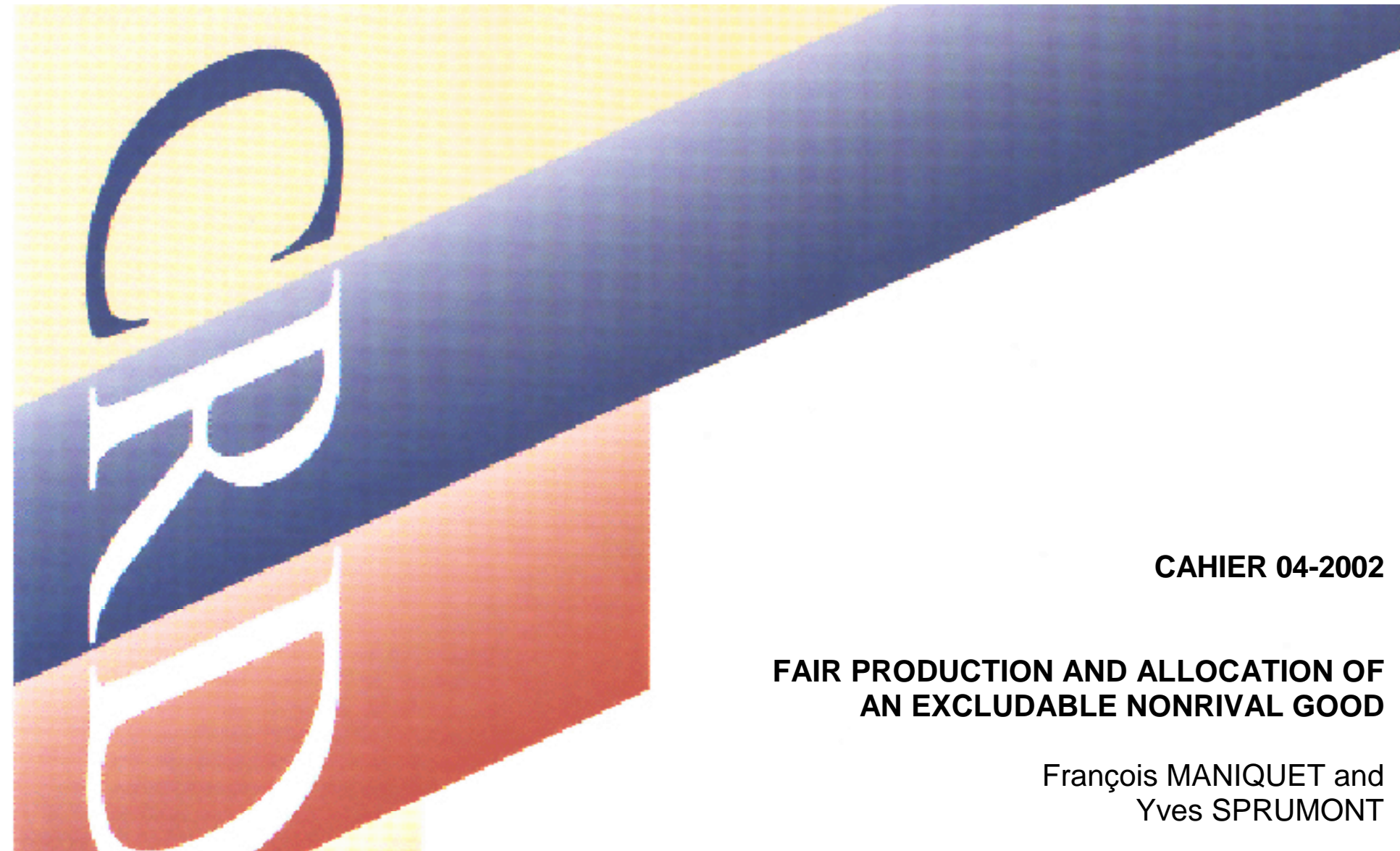

Centre de recherche et développement en économique

C.P. 6128, succursale Centre-ville Montréal QC H3C 3J7

Téléphone : (514) 343-6557

Télécopieur : (514) 343-5831

crde@crde.umontreal.ca

http://www. crde. umontreal.ca/

Université $\mathbf{t h}$ de Montréal 
CAHIER 04-2002

\title{
FAIR PRODUCTION AND ALLOCATION OF AN EXCLUDABLE NONRIVAL GOOD
}

\author{
François MANIQUET ${ }^{1}$ and Yves SPRUMONT ${ }^{2}$
}

1 Institute for Advanced Study, Princeton University, and F.N.R.S., University of Namur

2 Centre de recherche et développement en économique (C.R.D.E.) and Département de sciences économiques, Université de Montréal

February 2002

The authors thank seminar participants at the Institute for Advanced Study, and especially Massimo Morelli, for their comments and suggestions. Sprumont gratefully acknowledges financial support from the Social Sciences and Humanities Research Council of Canada and the Fonds pour la formation de chercheurs et l'aide à la recherche of Québec. 


\title{
RÉSUMÉ
}

Dans le contexte d'une économie à un bien privé et un bien non rival, nous montrons que trois principes normatifs simples forcent à classer les allocations en appliquant le critère maximin aux vecteurs de bien-être mesuré en termes du bien non rival.

Mots clés : équité, bien non rival, exclusion, ordres sociaux

\begin{abstract}
We study fairness in economies with one private good and one partially excludable nonrival good. A social ordering function determines for each profile of preferences an ordering of all conceivable allocations. We propose the following Free Lunch Aversion condition: if the private good contributions of two agents consuming the same quantity of the nonrival good have opposite signs, reducing that gap improves social welfare. This condition, combined with the more standard requirements of Unanimous Indifference and Responsiveness, delivers a form of welfare egalitarianism in which an agent's welfare at an allocation is measured by the quantity of the nonrival good that, consumed at no cost, would leave her indifferent to the bundle she is assigned.
\end{abstract}

Key words : fairness, excludable nonrival good, social orderings 


\section{Introduction}

We consider an environment where a private good can be used to produce a nonrival good. We assume that different agents may consume different quantities of the nonrival good: "partial exclusion" is possible. Examples of such goods include cable television programs and noncongested parks, roads, libraries, or sport facilities. The agents are endowed with (large) amounts of the private good, and they own the production technology in common. The problem we face is to choose how much nonrival good to produce, how much of it each agent is allowed to consume, and how much private good each agent is asked to contribute. We are interested in efficiency as well as equity.

The standard approach to this problem is to look for allocation rules. An allocation rule specifies which feasible allocations are the most desirable as a function of the parameters of the problem, namely, the production technology and the agents' preferences over bundles of private and nonrival goods. Contributions to that literature include Foley (1967), Mas-Colell (1980), Moulin (1987), Sprumont (1998). Quite naturally, all these papers impose Pareto efficiency. This immediately rules out exclusion: all agents should consume the entire production of the nonrival good.

An alternative approach is to look for social ordering functions. A (social ordering) function specifies a complete ranking of all conceivable allocations, feasible or not, as a function of the parameters of the problem. This paper deals with such social ordering functions. We are motivated by second-best considerations. In many circumstances, it is not sufficient to know which technologically feasible allocations are the most desirable. For instance, if an allocation has already been chosen and is viewed by all as a legitimate status quo, any recommended allocation should dominate this status quo (and, in addition, might have to stay sufficiently close to it). In the same vein, if the nonrival good production and consumption levels have been fixed and only individual contributions remain to be chosen, the most desirable allocation need again not be achievable. Lastly, but most importantly, achievable allocations may be restricted by incentive-compatibility constraints. In fact, the presence of such constraints is a primary motivation for considering allocations where agents are partially excluded from the consumption of the nonrival good: as Moulin (1994) demonstrates, allowing partial exclusion helps alleviate the free-rider problem.

To sum up, we believe that the set of achievable allocations is often very 
uncertain and may have almost any shape. It is therefore very useful, and perhaps even necessary, to determine a full ordering of the conceivable allocations so as to be able to make a best choice from virtually any set.

We start from three simple principles for ordering allocations. First, Unanimous Indifference: allocations that leave all agents indifferent should be equivalent. Second, Responsiveness: a social preference for an allocation over another should be preserved when all agents' upper contour sets at the better allocation shrink and their upper contour sets at the worse allocation expand. Third, Free Lunch Aversion: if two agents consume the same quantity of the nonrival good, but one contributes positively to its production whereas the other contributes negatively - thereby enjoying a "free lunch" -, a transfer of private good from the latter to the former agent should be regarded as a social improvement, provided that such a transfer does not reverse the signs of the agents' contributions.

We show that these three principles lead to a specific class of egalitarian, or maximin, social ordering functions: at all profiles, allocations must be ranked according to the welfare of the worst-off agent measured in terms of the nonrival good. The nonrival good measure of an agent's welfare at a given allocation is defined as the quantity of the nonrival good whose consumption for free would leave her indifferent to her actual bundle. A prominent social ordering function satisfying our three principles is the nonrival-welfare leximin function.

\section{Setup}

One nonrival good may be produced from one private good. There is a fixed finite set of agents, $N=\{1, \ldots, n\}$, with $n \geq 2$. We denote by $z_{i}=$ $\left(x_{i}, y_{i}\right) \in \mathbb{R} \times \mathbb{R}_{+}$agent $i$ 's consumption bundle. Here $x_{i}$ is the quantity of the private good that agent $i$ contributes to the production process: a positive value means that $i$ consumes less than her endowment in the private good, which is left unspecified. By $y_{i}$ we denote the quantity of the nonrival good that agent $i$ consumes. This formulation allows different agents to consume different quantities of the nonrival good: exclusion, complete or partial, is possible. At the same time, we allow for negative private good contributions: they might be useful to compensate for exclusion.

A preference for agent $i$ is a binary relation $R_{i}$ over $\mathbb{R} \times \mathbb{R}_{+}$which is complete, transitive, continuous, strictly decreasing in the private good con- 
tribution level $x_{i}$, strictly increasing in the nonrival good consumption level $y_{i}$, and convex. If $z_{i} \in \mathbb{R} \times \mathbb{R}_{+}, B\left(R_{i}, z_{i}\right)=\left\{z_{i}^{\prime} \in \mathbb{R} \times \mathbb{R}_{+} \mid z_{i}^{\prime} R_{i} z_{i}\right\}$ is the upper contour set of $R_{i}$ at $z_{i}$. The indifference and strict preference relations corresponding to $R_{i}$ are denoted by $I_{i}$ and $P_{i}$. The set of all preferences is denoted by $\mathcal{R}$. A (preference) profile is a list $R \in \mathcal{R}^{N}$.

An allocation is a vector $z=\left(z_{1}, \ldots, z_{n}\right) \in\left(\mathbb{R} \times \mathbb{R}_{+}\right)^{N}$. It is admissible for $R$ if there exists a production level $\bar{y}$ such that, for each $i \in N$,

$$
(0, \bar{y}) R_{i} z_{i} R_{i}(0,0)
$$

Thus, no agent prefers her bundle to the opportunity of consuming any quantity of the nonrival good for free, and everyone receives a non-negative share of the surplus generated by the production of the nonrival good. We let $Z_{i}\left(R_{i}\right)$ stand for the set of bundles $z_{i}$ satisfying (1) and we denote the set of admissible allocations for $R$ by $Z(R)$. If $i, j \in N$ are two distinct agents, $z_{-i}$ denotes the restriction of the allocation $z$ to $N \backslash\{i\}$ and $z_{-i j}$ is the restriction of $z$ to $N \backslash\{i, j\}$.

A social ordering for $R$ is a complete and transitive binary relation defined over $Z(R)$, the set of all admissible allocations for $R$. A social ordering function $\mathbf{R}$ assigns to each preference profile $R \in \mathcal{R}^{N}$ a social ordering $\mathbf{R}(R)$ for $R$. Thus, $z \mathbf{R}(R) z^{\prime}$ means that the allocation $z$ is at least as desirable as $z^{\prime}$ from a social viewpoint if the preference profile is $R$. Similarly, we use $\mathbf{I}(R)$ and $\mathbf{P}(R)$ to denote social indifference and strict social preference.

One feature of our setup calls for a word of explanation. In contrast to the literature on allocation rules, we make no assumption regarding technology. In fact, the production function relating the nonrival good to the private good contributions is left unspecified. The reason is that we are interested in defining social objectives rather than recommending choices directly. Since the social orderings that we discuss do not depend on the technology, there is no need to incorporate the latter in the model. Of course, the social choices implicitly recommended by a social ordering $\mathbf{R}(R)$ result from constrained maximization. Thus, if $c(y)$ were the cost of producing a quantity $y$ of the nonrival good, the recommended allocations would be those that maximize $\mathbf{R}(R)$ subject to the inequality $\sum_{i \in N} x_{i} \geq c\left(\max _{i \in N} y_{i}\right)$. We briefly return to this issue in the concluding section. 


\section{Axioms}

The rather uncontroversial idea that social preferences should agree with individual preferences whenever the latter happen to be unanimous admits several formulations. We consider the following three versions.

Unanimous Indifference. Let $R \in \mathcal{R}^{N}$ and $z, z^{\prime} \in Z(R)$. If $z_{i} I_{i} z_{i}^{\prime}$ for all $i \in N$, then $z \mathbf{I}(R) z^{\prime}$.

Unanimity. Let $R \in \mathcal{R}^{N}$ and $z, z^{\prime} \in Z(R)$. If $z_{i} R_{i} z_{i}^{\prime}$ for all $i \in N$, then $z \mathbf{R}(R) z^{\prime}$. If $z_{i} P_{i} z_{i}^{\prime}$ for all $i \in N$, then $z \mathbf{P}(R) z^{\prime}$.

Strong Pareto Principle. Let $R \in \mathcal{R}^{N}$ and $z, z^{\prime} \in Z(R)$. If $z_{i} R_{i} z_{i}^{\prime}$ for all $i \in N$, then $z \mathbf{R}(R) z^{\prime}$. If $z_{i} R_{i} z_{i}^{\prime}$ for all $i \in N$ and $z_{i} P_{i} z_{i}^{\prime}$ for some $i \in N$, then $z \mathbf{P}(R) z^{\prime}$.

The Strong Pareto Principle implies Unanimity, which in turns implies Unanimous Indifference; the converse implications do not hold. In what follows, we will only impose Unanimous Indifference. Combined with the two axioms defined below, Unanimous Indifference turns out to imply Unanimity.

Our next axiom, Responsiveness, is an inter-profile property requiring a form of robustness of the social ranking when individual preferences change. If an allocation $z$ is socially preferred to an allocation $z^{\prime}$, that social preference should be preserved when $z$ "moves upwards" in all agents' preferences whereas $z^{\prime}$ "moves downwards". Upwards and downwards moves are evaluated by looking at upper contour sets. Thus, Responsiveness requires that the social preference for $z$ over $z^{\prime}$ be preserved when all agents' upper contour sets at (the bundle they are assigned at) $z$ shrink whereas their upper contour sets at $z^{\prime}$ expand. Our axiom imposes this robustness on both the weak and the strict social preference relations.

Responsiveness. Let $R, R^{\prime} \in \mathcal{R}^{N}$ and $z, z^{\prime} \in Z(R) \cap Z\left(R^{\prime}\right)$. Suppose that for all $i \in N, B\left(R_{i}^{\prime}, z_{i}\right) \subseteq B\left(R_{i}, z_{i}\right)$ and $B\left(R_{i}^{\prime}, z_{i}^{\prime}\right) \supseteq B\left(R_{i}, z_{i}^{\prime}\right)$. Then, $\left\{z \mathbf{R}(R) z^{\prime}\right\} \Rightarrow\left\{z \mathbf{R}\left(R^{\prime}\right) z^{\prime}\right\}$ and $\left\{z \mathbf{P}(R) z^{\prime}\right\} \Rightarrow\left\{z \mathbf{P}\left(R^{\prime}\right) z^{\prime}\right\}$.

This property, which we borrow from Fleurbaey and Maniquet (1996), is reminiscent of several inter-profile robustness axioms that played a major role in social choice and implementation theory, such as Maskin's (1998) monotonicity. We refer to Fleurbaey and Maniquet (1996) and Le Breton and Weymark (2001) for a discussion of such properties. Responsiveness is appealing from an ethical point of view because it guarantees that unambiguous changes in individual preferences are reflected in the social ordering 
of allocations. As many other robustness axioms, it may also be defended on grounds of informational simplicity. Note in particular that once a strict social preference has been established between two allocations, it cannot be reversed by changes in preferences that leave the individual upper contour sets at those allocations unchanged.

Finally, we turn to our third axiom, Free Lunch Aversion. Consider a profile $R$ and an allocation at which two agents, $i$ and $j$, consume the same quantity of the nonrival good. Suppose that $i$ 's private good contribution is positive but $j$ 's contribution is negative. Since $j$ enjoys a "free lunch", a transfer of private good from $j$ to $i$ that does not reverse the signs of their contributions should be deemed to increase social welfare.

Free Lunch Aversion. Let $R \in \mathcal{R}^{N},(x, y),\left(x^{\prime}, y\right) \in Z(R)$, and $i, j \in N$. Suppose that $x_{-i j}=x_{-i j}^{\prime}, y_{i}=y_{j}$, and $x_{i}+x_{j}=x_{i}^{\prime}+x_{j}^{\prime}$. Then, $\left\{x_{j}<x_{j}^{\prime} \leq\right.$ $\left.0 \leq x_{i}^{\prime}<x_{i}\right\} \Rightarrow\left\{\left(x^{\prime}, y\right) \mathbf{P}(R)(x, y)\right\}$.

This axiom is directly inspired by Moulin's (1987) No Private Transfers condition. Moulin's condition, which applies to allocation rules rather than social ordering functions, requires that everyone contribute a non-negative quantity of the private good to finance the production of the nonrival good. In our axiom, the proviso that both agents consume the same quantity of the nonrival good is crucial. Indeed, as exclusion is possible, it may be fair to let people contribute less than others if they also consume less of the nonrival good. Even a negative contribution may be sensible, if it is the required compensation for being partially excluded.

\section{The result}

We now state and discuss our result. It concerns a form of welfare egalitarianism under which an agent's welfare is measured in terms of the nonrival good, that is, by the quantity of nonrival good which, consumed for free, would make her indifferent to the bundle she receives.

For each $R_{i} \in \mathcal{R}$ and $z_{i} \in Z_{i}\left(R_{i}\right)$, there is a unique level of the nonrival good, $y_{i}^{0} \in \mathbb{R}_{+}$, such that $z_{i} I_{i}\left(0, y_{i}^{0}\right)$. We may therefore define the numerical welfare representation function $u\left(R_{i},.\right): Z_{i}\left(R_{i}\right) \rightarrow \mathbb{R}_{+}$by letting

$$
u\left(R_{i}, z_{i}\right)=y_{i}^{0} \Leftrightarrow z_{i} I_{i}\left(0, y_{i}^{0}\right) .
$$


The number $u\left(R_{i}, z_{i}\right)$ is agent $i$ 's nonrival good welfare level at bundle $z_{i}$.

A social ordering function $\mathbf{R}$ is a nonrival-good-welfare maximin function if, at any profile, the ordering of allocations it prescribes is consistent with the application of the maximin criterion to the nonrival good welfare levels generated by these allocations. More precisely: for any $R \in \mathcal{R}^{N}$ and $z, z^{\prime} \in$ $Z(R)$

$$
\min _{i \in N} u\left(R_{i}, z_{i}\right)>\min _{i \in N} u\left(R_{i}, z_{i}^{\prime}\right) \Rightarrow z \mathbf{P}(R) z^{\prime} .
$$

A prominent example is the nonrival-good-welfare leximin function $\mathbf{R}_{L}$. Let $\succcurlyeq_{L}$ denote the usual leximin ordering of $\mathbb{R}_{+}^{N}$ : for any $w, w^{\prime} \in \mathbb{R}_{+}^{N}, w \succcurlyeq w^{\prime}$ if and only if the smallest coordinate of $w$ is greater than the smallest coordinate of $w^{\prime}$, or they are equal but the second smallest coordinate of $w$ is greater than the second smallest coordinate of $w^{\prime}$, and so on. The social ordering function $\mathbf{R}_{L}$ ranks the admissible allocations for any given preference profile by applying the leximin ordering to the corresponding vectors of nonrival good welfare levels: for any $R \in \mathcal{R}^{N}$ and $z, z^{\prime} \in Z(R)$,

$$
z \mathbf{R}_{L}(R) z^{\prime} \Leftrightarrow\left(u\left(R_{1}, z_{1}\right), \ldots, u\left(R_{n}, z_{n}\right)\right) \succcurlyeq_{L}\left(u\left(R_{1}, z_{1}^{\prime}\right), \ldots, u\left(R_{n}, z_{n}^{\prime}\right)\right) .
$$

This social ordering function meets all of the axioms considered in Section 3. Conversely, these axioms force us to use a nonrival-good-welfare maximin function.

Theorem.

i) The nonrival-good-welfare leximin function $\mathbf{R}_{L}$ satisfies the Strong Pareto Principle, Responsiveness, and Free Lunch Aversion.

ii) Every social ordering function $\mathbf{R}$ satisfying Unanimous Indifference, Responsiveness, and Free Lunch Aversion is a nonrival-good-welfare maximin function satisfying Unanimity.

A few comments are in order.

1) At the risk of stressing the obvious, we emphasize that the axioms and the social ordering functions appearing in our theorem are ordinal. The information used to define them is entirely contained in the agents' preferences, which are simply orderings over consumption bundles: no utility information is available in our model. Our result is therefore fundamentally different from the various classical characterizations of the maximin and leximin social welfare orderings surveyed, for instance, in d'Aspremont and Gevers (2001). In 
that literature, individual utilities are given and social welfare orderings are constructed over vectors of individual utilities.

Having stressed that point, it appears that the contribution of (the second part of) our theorem is really twofold. Not only do our axioms lead us to rank allocations by applying the maximin criterion to vectors of corresponding welfare levels, they also force us to use a specific welfare representation of preferences, namely, the nonrival-good-welfare representation.

2) (nonrival-good-)welfare maximin functions are radically averse to (nonrival-good-)welfare inequalities: social welfare may be improved by an arbitrarily small increase in the welfare of the worst-off agent obtained at the cost of possibly huge welfare losses to the others.

It is interesting and somewhat surprising that our axioms deliver such an extreme conclusion. Clearly, Free Lunch Aversion expresses a form of welfare inequality aversion. But it is a very mild one. Indeed, Free Lunch Aversion implies that reducing the nonrival-good-welfare gap between two agents improves social welfare only when two very specific conditions are met: the agents must consume the same quantity of the nonrival good, and the welfare gap reduction must be obtained by a mere private good transfer from one to the other. This should be contrasted with much more radical axioms of welfare inequality aversion used in characterizations of the classical leximin social welfare ordering over utility space, such as Hammond's (1976) equity axiom.

Unanimous Indifference and Responsiveness, however, happen to complement Free Lunch Aversion remarkably. Starting with an allocation where two agents enjoy different nonrival good welfare levels, Unanimous Indifference allows us, by "sliding along their indifference curves", to find socially equivalent allocations where their consumptions of the nonrival good are equal and their private good contributions have opposite signs. By Free Lunch Aversion, any welfare inequality reduction resulting from a sufficiently small private good transfer from the better-off to the worse-off agent improves social welfare. It turns out that Responsiveness forces us to extend this conclusion to welfare inequality reductions that involve an arbitrarily small welfare gain against a large welfare loss, thereby reaching the maximin criterion.

3) The axioms in statement ii) of our theorem are independent.

The function assigning to each preference profile the social ordering according to which all allocations are equivalent trivially satisfies Unanimous Indifference and Responsiveness, but violates Free Lunch Aversion. The 
nonrival-good-welfare utilitarian function $\mathbf{R}_{U}$ defined by $z \mathbf{R}_{U}(R) z^{\prime}$ if and only if $\sum_{i \in N} u\left(R_{i}, z_{i}\right) \geq \sum_{i \in N} u\left(R_{i}, z_{i}^{\prime}\right)$ is another example; note that it even satisfies the Strong Pareto Principle.

The function $\mathbf{R}_{V}$ defined by $(x, y) \mathbf{R}_{V}(R)\left(x^{\prime}, y^{\prime}\right)$ if and only if $\sum_{i \in N}\left|x_{i}\right| \leq$ $\sum_{i \in N}\left|x_{i}^{\prime}\right|$ satisfies Free Lunch Aversion and Responsiveness, but not Unanimous Indifference.

Finally, consider the following social ordering function $\mathbf{R}_{L, U}$. On those preference profiles where all agents have strictly convex preferences that are additively separable and linear in the nonrival good, $\mathbf{R}_{L, U}$ coincides with $\mathbf{R}_{U}$. On all other profiles, $\mathbf{R}_{L, U}$ coincides with $\mathbf{R}_{L}$. This function satisfies Unanimous Indifference, Free Lunch Aversion (strict convexity guarantees that the sum of utilities after a "free lunch transfer" be strictly larger than before the transfer), but not Responsiveness.

4) Our theorem is not quite a complete characterization.

Regarding statement i), $\mathbf{R}_{L}$ is not the only social ordering function satisfying the Strong Pareto Principle, Responsiveness, and Free Lunch Aversion. Other examples include functions that agree with $\mathbf{R}_{L}$ whenever the latter does not declare a tie between two allocations, but break such ties according to an a priori ordering of the agents. For instance, let $\succcurlyeq_{l}$ be the lexicographic ordering of $\mathbb{R}_{+}^{N}$ corresponding to the natural ordering of the agents and define $z \mathbf{R}_{L, l}(R) z^{\prime}$ if and only if either i) $z \mathbf{P}_{L}(R) z^{\prime}$ or ii) $z \mathbf{I}_{L}(R) z^{\prime}$ and $\left(u\left(R_{1}, z_{1}\right), \ldots, u\left(R_{n}, z_{n}\right)\right) \succcurlyeq_{l}\left(u\left(R_{1}, z_{1}^{\prime}\right), \ldots, u\left(R_{n}, z_{n}^{\prime}\right)\right)$. The social ordering function $\mathbf{R}_{L, l}$ satisfies the Strong Pareto Principle, Free Lunch Aversion and Responsiveness.

Regarding statement ii), not all nonrival-good-welfare maximin functions respecting Unanimity satisfy Responsiveness or Free Lunch Aversion. For instance, the function $\mathbf{R}_{M}$ defined by $z \mathbf{R}_{M}(R) z^{\prime} \Leftrightarrow \min _{i \in N} u\left(R_{i}, z_{i}\right) \geq$ $\min _{i \in N} u\left(R_{i}, z_{i}^{\prime}\right)$ violates Free Lunch Aversion. The reader may easily modify this example to construct nonrival-good-welfare maximin functions that satisfy the Strong Pareto Principle but violate both Responsiveness and Free Lunch Aversion.

5) In addition to the properties stated in our theorem, the nonrival-goodwelfare leximin function satisfies many other axioms that may be adapted from social choice theory to our setting. For instance, it is independent of the feasible set, in the sense that the ordering of allocations recommended at any preference profile does not vary with the technology of production of the nonrival good (see Fleurbaey and Maniquet (2001) for a discussion of such 
independence properties). It also possesses properties of separability similar to those enjoyed by the classical leximin social welfare ordering over utility space (see d'Aspremont and Gevers (2001) for a survey).

\section{Proof of the result}

Unanimity is the conjunction of two conditions which we find useful to separate and name as follows.

Unanimous Preference. Let $R \in \mathcal{R}^{N}$ and $z, z^{\prime} \in Z(R)$. If $z_{i} R_{i} z_{i}^{\prime}$ for all $i \in N$, then $z \mathbf{R}(R) z^{\prime}$.

Unanimous Strict Preference. Let $R \in \mathcal{R}^{N}$ and $z, z^{\prime} \in Z(R)$. If $z_{i} P_{i} z_{i}^{\prime}$ for all $i \in N$, then $z \mathbf{P}(R) z^{\prime}$.

We proceed by proving two lemmata.

Lemma 1. If a social ordering function $\mathbf{R}$ satisfies Unanimous Indifference and Responsiveness, then it satisfies Unanimous Preference.

Proof. Let $\mathbf{R}$ satisfy Unanimous Indifference and Responsiveness. Suppose that, contrary to the claim, $\mathbf{R}$ violates Unanimous Preference. There must exist a profile $R \in \mathcal{R}^{N}$, two allocations $z^{1}, z^{2} \in Z(R)$, and an agent $i \in N$ such that $z_{i}^{2} P_{i} z_{i}^{1}, z_{-i}^{2}=z_{-i}^{1}$, and $z^{1} \mathbf{P}(R) z^{2}$. Because of Responsiveness, we may assume that $B_{i}\left(R_{i}, z_{i}^{1}\right)$ is strictly convex, that is, $\lambda z_{i}^{1}+(1-\lambda) z_{i}$ $P_{i} z_{i}^{1}$ whenever $z_{i} I_{i} z_{i}^{1}, z_{i} \neq z_{i}^{1}$, and $0<\lambda<1$.

As illustrated on Figure 1 , choose $z_{i}^{3}=\left(x_{i}^{3}, y_{i}^{3}\right)$ such that $z_{i}^{3} I_{i} z_{i}^{1}$ and $y_{i}^{3}>y_{i}^{1}, y_{i}^{2}$. Let $C$ be the convex hull of $\left.\left\{\left(x_{i}, y_{i}\right) \in B\left(R_{i}, z_{i}^{1}\right) \mid y_{i} \geq y_{i}^{3}\right)\right\} \cup$ $B\left(R_{i}, z_{i}^{2}\right)$ and denote by $\partial C$ the strict south-east frontier of $C$, that is, $\partial C=$ $\left\{\left(x_{i}, y_{i}\right) \in C \mid\left(x_{i}^{\prime}, y_{i}^{\prime}\right)=\left(x_{i}, y_{i}\right)\right.$ for all $\left(x_{i}^{\prime}, y_{i}^{\prime}\right) \in C$ such that $x_{i}^{\prime} \geq x_{i}$ and $y_{i}^{\prime} \leq$ $\left.y_{i}\right\}$. By construction, there exists $z_{i}^{4} \in \partial C$ such that $z_{i}^{4} I_{i} z_{i}^{2}$. By Unanimous Indifference, $\left(z_{i}^{3}, z_{-i}^{1},\right) \mathbf{P}(R)\left(z_{i}^{4}, z_{-i}^{2}\right)$.

Now, construct $R_{i}^{\prime} \in \mathcal{R}$ such that $B\left(R_{i}^{\prime}, z_{i}^{3}\right)=C$ and $B\left(R_{i}^{\prime}, z_{i}^{1}\right) \supseteq B\left(R_{i}, z_{i}^{1}\right)$. Such a construction is possible because $B_{i}\left(R_{i}, z_{i}^{1}\right)$ is strictly convex. Since $z_{i}^{4} \in \partial C$, we have $z_{i}^{3} I_{i}^{\prime} z_{i}^{4}$. But by Responsiveness, $\left(z_{i}^{3}, z_{-i}^{1}\right) \mathbf{P}\left(R^{\prime}\right)\left(z_{i}^{4}, z_{-i}^{2}\right)$, contradicting Unanimous Indifference.

Lemma 2. If a social ordering function $\mathbf{R}$ satisfies Unanimous Preference and Free Lunch Aversion, then it satisfies Unanimous Strict Preference. 
Proof. Let $\mathbf{R}$ satisfy Unanimous Preference and Free Lunch Aversion. Suppose now, by way of contradiction, that $\mathbf{R}$ violates Unanimous Strict Preference. Let $R \in \mathcal{R}^{N}$ and $z^{1}, z^{2} \in Z(R)$ be such that $z_{i}^{2} P_{i} z_{i}^{1}$ for all $i \in N$ and

$$
z^{1} \mathbf{R}(R) z^{2} .
$$

Without loss of generality, assume that $u\left(R_{1}, z_{1}^{1}\right) \geq u\left(R_{i}, z_{i}^{1}\right)$ for all $i \in N$. It follows that $u\left(R_{1}, z_{1}^{2}\right)>u\left(R_{i}, z_{i}^{1}\right)$ for all $i \in N$. As illustrated on Figure 2, we can therefore construct $z_{1}^{3}=\left(x_{1}^{3}, y^{3}\right)$ and $z_{2}^{3}=\left(x_{2}^{3}, y^{3}\right)$ such that $x_{1}^{3}<$ $0<x_{2}^{3}, z_{1}^{3} I_{1} z_{1}^{2}$, and $z_{2}^{3} I_{2} z_{2}^{1}$. By continuity of $R_{1}$ and $R_{2}$, we can find $\varepsilon>0$ small enough to guarantee that $x_{1}^{3}+\varepsilon<0<x_{2}^{3}-\varepsilon,\left(x_{1}^{3}+\varepsilon, y^{3}\right) P_{1} z_{1}^{1}$, and $z_{2}^{2} P_{2}\left(x_{2}^{3}-\varepsilon, y^{3}\right)$.

By Free Lunch Aversion, Unanimous Indifference, and Unanimous Preference, $\left(\left(x_{1}^{3}+\varepsilon, y^{3}\right),\left(x_{2}^{3}-\varepsilon, y^{3}\right), z_{-12}^{1}\right) \mathbf{P}(R)\left(z_{1}^{3}, z_{2}^{3}, z_{-12}^{1}\right) \mathbf{I}(R)\left(z_{1}^{3}, z_{-1}^{1}\right) \mathbf{R}(R)$ $z^{1}$. Recalling (2), we conclude that $\left(\left(x_{1}^{3}+\varepsilon, y^{3}\right),\left(x_{2}^{3}-\varepsilon, y^{3}\right), z_{-12}^{1}\right) \mathbf{P}(R) z^{2}$, contradicting Unanimous Preference.

Proof of the Theorem. The straightforward proof that $\mathbf{R}_{L}$ satisfies the Strong Pareto Principle and Responsiveness is left to the reader. To check Free Lunch Aversion, let $R \in \mathcal{R}^{N},(x, y),\left(x^{\prime}, y\right) \in Z(R)$, and $i, j \in N$. Suppose that $x_{-i j}=x_{-i j}^{\prime}, y_{i}=y_{j}, x_{i}+x_{j}=x_{i}^{\prime}+x_{j}^{\prime}$, and $x_{j}<x_{j}^{\prime} \leq 0 \leq$ $x_{i}^{\prime}<x_{i}$. Because preferences are strictly decreasing in private good contributions, $\left(0, y_{i}\right) R_{i}\left(x_{i}^{\prime}, y_{i}\right) P_{i}\left(x_{i}, y_{i}\right)$ and $\left(x_{j}, y_{j}\right) P_{j}\left(x_{j}^{\prime}, y_{j}\right) R_{j}\left(0, y_{j}\right)$. Therefore $u\left(R_{i},\left(x_{i}, y_{i}\right)\right)<u\left(R_{i},\left(x_{i}^{\prime}, y_{i}\right)\right) \leq y_{i}=y_{j} \leq u\left(R_{j},\left(x_{j}^{\prime}, y_{j}\right)\right)<u\left(R_{j},\left(x_{j}, y_{j}\right)\right)$ and, hence, $\left(u\left(R_{1},\left(x_{1}^{\prime}, y_{1}\right)\right), \ldots, u\left(R_{n},\left(x_{n}^{\prime}, y_{n}\right)\right)\right) \succcurlyeq_{L}\left(u\left(R_{1},\left(x_{1}, y_{1}\right)\right), \ldots\right.$, $\left.u\left(R_{n},\left(x_{n}, y_{n}\right)\right)\right)$, as desired.

Conversely, let $\mathbf{R}$ satisfy Unanimous Indifference, Responsiveness, and Free Lunch Aversion. By Lemmata 1 and 2, $\mathbf{R}$ satisfies Unanimity. It remains to be shown that $\mathbf{R}$ is a nonrival-good-welfare maximin function. Suppose, by way of contradiction, that there exist $R \in \mathcal{R}^{N}$ and $z, z^{\prime} \in Z(R)$ such that

$$
\min _{i \in N} u\left(R_{i}, z_{i}\right)<\min _{i \in N} u\left(R_{i}, z_{i}^{\prime}\right)
$$

and

$$
z \mathbf{R}(R) z^{\prime}
$$

Define $M$ to be the unique subset of $N$ such that

$$
u\left(R_{i}, z_{i}\right)<\min _{i \in N} u\left(R_{i}, z_{i}^{\prime}\right) \leq u\left(R_{j}, z_{j}\right) \text { for all } i \in M \text { and all } j \in N \backslash M,
$$


and let $m$ denote the cardinality of $M$. By (3), $m>0$. We may further assume that $m<n$ because (4) and (5) directly conflict with Unanimous Strict Preference if $m=n$. We will construct a preference profile $R^{\prime}$, two allocations $q, q^{\prime} \in Z\left(R^{\prime}\right)$, and a set $M^{\prime}$ of cardinality $m+1$ such that

$$
u\left(R_{i}^{\prime}, q_{i}\right)<\min _{i \in N} u\left(R_{i}^{\prime}, q_{i}^{\prime}\right) \leq u\left(R_{j}^{\prime}, q_{j}\right) \text { for all } i \in M^{\prime} \text { and all } j \in N \backslash M^{\prime}
$$

and

$$
q \mathbf{R}\left(R^{\prime}\right) q^{\prime}
$$

Repeating the construction $n-m$ times eventually yields a contradiction to Unanimous Strict Preference.

The construction is illustrated on Figure 3. Assume, without loss of generality, that $1 \in M$ and $2 \in N \backslash M$. Assume, furthermore, that

$$
u\left(R_{1}, z_{1}\right)<u\left(R_{2}, z_{2}^{\prime}\right)=\min _{i \in N} u\left(R_{i}, z_{i}^{\prime}\right)<u\left(R_{1}, z_{1}^{\prime}\right)<u\left(R_{2}, z_{2}\right) .
$$

This entails no loss of generality: if (8) does not hold, there exist $t, t^{\prime} \in Z(R)$ such that $u\left(R_{i}, z_{i}\right) \leq u\left(R_{i}, t_{i}\right)$ and $u\left(R_{i}, z_{i}^{\prime}\right) \geq u\left(R_{i}, t_{i}^{\prime}\right)$ for all $i \in N$, and the conditions obtained from (5) and (8) by replacing $z$ and $z^{\prime}$ with $t$ and $t^{\prime}$ hold. By Unanimity and (4), $t \mathbf{R}(R) z \mathbf{R}(R) z^{\prime} \mathbf{R}(R) t^{\prime}$, hence, $t \mathbf{R}(R) t^{\prime}$, and we need only replace $z$ and $z^{\prime}$ with $t$ and $t^{\prime}$ in the argument below.

To alleviate notation, let us write $u\left(R_{i}, z_{i}\right)=u_{i}$ and $u\left(R_{i}, z_{i}^{\prime}\right)=u_{i}^{\prime}$ for $i=1,2$. By definition of $u\left(R_{i},.\right)$, and Unanimous Indifference, (4) yields

$$
\left(\left(0, u_{1}\right),\left(0, u_{2}\right), z_{-12}\right) \mathbf{R}(R)\left(\left(0, u_{1}^{\prime}\right),\left(0, u_{2}^{\prime}\right), z_{-12}^{\prime}\right) .
$$

Our continuity and strict monotonicity assumptions on preferences guarantee that for any sufficiently small $\varepsilon>0$ there exist $x_{1}(\varepsilon)>0$ and $x_{2}(\varepsilon)<0$ such that

$$
\begin{aligned}
& \left(x_{1}(\varepsilon), u_{2}-\varepsilon\right) I_{1}\left(0, u_{1}\right), \\
& \left(x_{2}(\varepsilon), u_{2}-\varepsilon\right) I_{2}\left(0, u_{2}\right),
\end{aligned}
$$

and $x_{1}(\varepsilon)+x_{2}(\varepsilon)>0$. Let us choose $\varepsilon>0$ small enough to also guarantee that $u_{2}^{\prime}<u_{2}-\varepsilon$ and that the quantity $y(\varepsilon)$ such that $(0, y(\varepsilon)) I_{1}\left(x_{1}(\varepsilon)+\right.$ $\left.x_{2}(\varepsilon), u_{2}-\varepsilon\right)$ is strictly between $u_{1}$ and $u_{2}^{\prime}$. 
By continuity and strict monotonicity of preferences again, there exist $y^{\prime}(\varepsilon), x_{1}^{\prime}(\varepsilon)>0$, and $x_{2}^{\prime}(\varepsilon)<0$ such that

$$
\begin{aligned}
& \left(x_{1}^{\prime}(\varepsilon), y^{\prime}(\varepsilon)\right) I_{1}\left(x_{1}(\varepsilon)+x_{2}(\varepsilon), u_{2}-\varepsilon\right), \\
& \left(x_{2}^{\prime}(\varepsilon), y^{\prime}(\varepsilon)\right) I_{2}\left(0, u_{2}^{\prime}\right),
\end{aligned}
$$

and $u_{1}<y(\varepsilon)<y^{\prime}(\varepsilon)<u_{2}^{\prime}$.

Next, choose $\varepsilon^{\prime}>0$ small enough to ensure that $x_{2}^{\prime}(\varepsilon)+\varepsilon^{\prime}<0<x_{1}^{\prime}(\varepsilon)-2 \varepsilon^{\prime}$ and $\left(0, u_{2}\right) P_{2}\left(x_{2}^{\prime}(\varepsilon)-\varepsilon^{\prime}, y^{\prime}(\varepsilon)\right)$. Construct a preference $R_{2}^{\prime}$ such that

$$
\begin{gathered}
B\left(R_{2}^{\prime},\left(0, u_{2}^{\prime}\right)\right)=B\left(R_{2},\left(0, u_{2}^{\prime}\right)\right), \\
\left(x_{2}^{\prime}(\varepsilon)-\varepsilon^{\prime}, y^{\prime}(\varepsilon)\right) I_{2}^{\prime}\left(0, u_{2}-\varepsilon\right), \\
\left(x_{2}(\varepsilon), u_{2}-\varepsilon\right) I_{2}^{\prime}\left(0, u_{2}\right), \\
B\left(R_{2}^{\prime},\left(0, u_{2}\right)\right) \subseteq B\left(R_{2},\left(0, u_{2}\right)\right) .
\end{gathered}
$$

Note that $\left(0, u_{2}\right) P_{2}^{\prime}\left(x_{2}^{\prime}(\varepsilon)+\varepsilon^{\prime}, y^{\prime}(\varepsilon)\right)$.

Let $R_{i}^{\prime}=R_{i}$ for all $i \in N \backslash 2$. Using successively Free Lunch Aversion, Unanimous Indifference, Free Lunch Aversion again, Unanimous Indifference again, and Responsiveness along with (9), we find the following chain of social preferences:

$$
\begin{aligned}
& \quad\left(\left(x_{1}^{\prime}(\varepsilon)-2 \varepsilon^{\prime}, y^{\prime}(\varepsilon)\right),\left(x_{2}^{\prime}(\varepsilon)+\varepsilon^{\prime}, y^{\prime}(\varepsilon)\right), z_{-12}\right) \\
& \mathbf{P}\left(R^{\prime}\right)\left(\left(x_{1}^{\prime}(\varepsilon), y^{\prime}(\varepsilon)\right),\left(x_{2}^{\prime}(\varepsilon)-\varepsilon^{\prime}, y^{\prime}(\varepsilon)\right), z_{-12}\right) \\
& \mathbf{I}\left(R^{\prime}\right)\left(\left(x_{1}(\varepsilon)+x_{2}(\varepsilon), u_{2}-\varepsilon\right),\left(0, u_{2}-\varepsilon\right), z_{-12}\right) \\
& \left.\mathbf{P}\left(R^{\prime}\right)\left(\left(x_{1}(\varepsilon), u_{2}-\varepsilon\right),\left(x_{2}(\varepsilon), u_{2}-\varepsilon\right)\right), z_{-12}\right) \\
& \mathbf{I}\left(R^{\prime}\right)\left(\left(0, u_{1}\right),\left(0, u_{2}\right), z_{-12}\right) \\
& \mathbf{R}\left(R^{\prime}\right)\left(\left(0, u_{1}^{\prime}\right),\left(0, u_{2}^{\prime}\right), z_{-12}^{\prime}\right) .
\end{aligned}
$$

Denote by $q$ and $q^{\prime}$ the first and last allocations in this chain, and define $M^{\prime}=M \cup\{2\}$. We have $q \mathbf{R}\left(R^{\prime}\right) q^{\prime}$, which is (7). By construction, $u\left(R_{1}^{\prime}, q_{1}\right)<$ $u\left(R_{2}^{\prime}, q_{2}\right)<u\left(R_{2}^{\prime}, q_{2}^{\prime}\right)=\min _{i \in N} u\left(R_{i}^{\prime}, q_{i}^{\prime}\right)$ and of course $q_{-12}=z_{-12}$ and $q_{-12}^{\prime}=$ $z_{-12}^{\prime}$. Therefore (5) implies $u\left(R_{i}^{\prime}, q_{i}\right)<\min _{i \in N} u\left(R_{i}^{\prime}, q_{i}^{\prime}\right) \leq u\left(R_{j}^{\prime}, q_{j}\right)$ for all $i \in M^{\prime}$ and all $j \in N \backslash M^{\prime}$, which is just (6). 


\section{Concluding comments}

Our axiomatic analysis has led us to recommend a particular social ordering function, the so-called nonrival-good-welfare leximin function. While our result concerns the definition of social objectives, it does provide normative guidelines for actual social choices. Given a preference profile $R$ and a suitably restricted cost function $c$, maximizing the social ordering $\mathbf{R}_{L}(R)$ subject to the technological constraint $\sum_{i \in N} x_{i} \geq c\left(\max _{i \in N} y_{i}\right)$ yields exactly the egalitarian-equivalent allocations advocated by Moulin (1987).

Incidentally, it is noteworthy that the technological constraint just stated is the only reason that really forces us to interpret the $y$ good as a nonrival good. Since this constraint is not part of our formal model, we remain free to regard the $y$ good as a private good and to reinterpret our axioms and our theorem in the context of production and cost-sharing of a private good. In such a context, the recommended choices would result from maximization subject to the alternative technological constraint $\sum_{i \in N} x_{i} \geq c\left(\sum_{i \in N} y_{i}\right)$. We focused on the nonrival good interpretation because we find the Free Lunch Aversion axiom particularly appealing in that context.

Finally, our result provides a systematic tool for recommending secondbest choices. Indeed, we may search for allocations that maximize the social ordering prescribed by the leximin function subject to any list of constraints that may be relevant, including incentive-compatibility requirements. We leave the study of such second-best allocation rules for future research.

\section{References}

d'Aspremont, C. and L. Gevers (2001), "Social welfare functionals and interpersonal comparability", in K. J. Arrow, A. K. Sen and K. Suzumura (Eds), Handbook of Social Choice and Welfare, Vol II, Ch 10, North-Holland: Amsterdam, forthcoming.

Fleurbaey, M. and F. Maniquet (1996), "Utilitarianism versus fairness in welfare economics", in Salles, M. and J. Weymark (eds) Justice, political liberalism and utilitarianism: themes from Harsanyi and Rawls, Cambridge: Cambridge University Press, forthcoming.

Fleurbaey, M. and F. Maniquet (2001), "Fair social orderings", mimeo. 
Foley, D. (1967), "Resource allocation and the public sector", Yale Economic Essays 7, 45-98.

Hammond, P. (1976), "Equity, Arrow's conditions and Rawls' difference principle", Econometrica 44, 793-804.

Le Breton, M. and J. Weymark (2001), "Arrovian social choice theory on economic models", in K. J. Arrow, A. K. Sen and K. Suzumura (Eds), Handbook of Social Choice and Welfare, Vol II, Ch 16, North-Holland: Amsterdam, forthcoming.

Mas-Colell, A. (1980), "Remarks on the game-theoretic analysis of a simple distribution of surplus problem", International Journal of Game Theory 9, 125-140.

Maskin, E. (1998), "Nash equilibrium and welfare optimality", Review of Economic Studies 66, 23-38.

Moulin, H. (1987), "Egalitarian-equivalent cost sharing of a public good", Econometrica 55, 963-976.

Moulin, H. (1994), "Serial cost sharing of excludable public goods", Review of Economic Studies 61, 305-325.

Sprumont, Y. (1998), "Equal factor equivalence in economies with multiple public goods", Social Choice and Welfare 15, 543-558. 